\title{
Youth Data Literacy: Teen Perspectives on Data Created with Social Media and Mobile Devices
}

\author{
Amelia Acker \\ University of Texas, Austin \\ School of Information \\ aacker@ischool.utexas.edu
}

\author{
Leanne Bowler \\ University of Pittsburgh \\ School of Computing and Information \\ lbowler@pitt.edu
}

\begin{abstract}
This paper examines how American teens conceptualize the term "data" in the context of social and mobile media like Instagram and Snapchat, or text messaging and cell phone video. Using interview and ethnographic data from a series of interviews with teens, 11 to 18 held in Pittsburgh, Pennsylvania public libraries over 2016-2017, we report findings about how teens learn about data through the use of social media platforms, the creation of mobile media, and the ownership of mobile devices; the implications that using networked platforms and wireless technology has for contemporary understandings of data literacy; and finally, what this means for teaching and researching the acquisition of data skills. The paper presents findings about how teens learn and acquire knowledge about the interactive and social processes of the data life cycle in public spaces and in online platforms, particularly learning about data awareness through sharing, aging, and owning mobile computing devices.
\end{abstract}

\section{Introduction}

Networked digital media technologies, from gaming stations to camera phones, have complicated the information landscape for members of the $21^{\text {st }}$ century, including what it means to grow up, from childhood to adulthood. Increasingly, next generation wireless networks ( $3 \mathrm{G}, 4 \mathrm{G}, \mathrm{LTE})$ that provide access to mobile broadband internet and high rates of mobile saturation around the world are shifting what it means to be a "data subject" in a networked era [31, 37]. Social network technologies that leverage the alwayson possibilities of next generation mobile networks have transformed the possibilities of being tethered to groups of people, and places such as home and work [14]. By constructing "networked publics" social media platforms such as Facebook or Snapchat have engendered new kinds of communities, modes of participation [12], ways of being private and negotiating public personas online. Contemporary understandings of the data lifecycle are tethered to the management of born digital data [8], yet social media platforms, pervasive wireless networks and cheap mobile devices now shape the data life cycleincreasingly the data we create is both born digital and born networked [2].

Given the rapid emergence of social media in the past decade, broadband internet access, and high rates of mobile device ownership in the United States, we ask what it means to grow up in a world where having a mobile device, multiple user accounts, and creating data traces is part of being a student, child, family member, and friend. How does owning mobile devices and creating born networked data collections influence today's networked youth [1]? How does it change the information services young people use to learn or understand data contexts and how it impacts our lives? In this paper, we report on early findings from a twoyear research study, entitled "Exploring Data Worlds at the Public Library" that investigates ways of supporting teens in public libraries to gain a sophisticated understanding of the data life cycle, including its creation, collection, aggregation, and flows of data across wireless devices and social media platforms, amongst other information infrastructures. In the paper that follows we describe some preliminary findings gathered from interviews and workshops with teens that took place in public libraries in Pittsburgh, Pennsylvania in late 2016 and early 2017. As information scientists using qualitative, interpretive methods, we are interested in gathering empirical observations about youth's understanding of data in public and informal contexts where information services, technology, and facilitators such as young adult librarians are present. Findings and outcomes of this long-term research will inform future data literacy programming and education curriculum for 
information professionals teaching data skills in a variety of contexts.

In order to get out what it means to be a teen and growing up as a data subject in this moment, we need to reveal what young people already know and believe about data as a concept, discover what perceptions they bring to definitions of data, and understanding themselves as agents in data lifecycles in their everyday lives. Herein we focus on teens perceptions and awareness of data, revealing their beliefs in relation to the nature of data in platforms and in devices, such as mobile phones or school-issued tablets, exploring how these devices relate to their knowledge of where data lives and how it flows through networks and across information infrastructure. What do teens think about digital data? Does it change as they get older and approach adulthood? How do they learn and acquire understandings of it through social media use, mobile apps, and device ownership? What do they understand about how data is created, used in different applications and for different purposes, where it is stored and how it is transmitted? And further, who owns aggregations of data and who has access to it? The answers to these questions provide insight into how teens acquire an awareness and sense of agency needed to assert control over data creation and collection contexts, and importantly, how we might shape future skills acquisition, measure learning outcomes and design informal learning experiences.

These findings are an outcome of an ongoing ethnographic study of teens and young adult (YA) librarians in public libraries and youth programming in public libraries, designed to gather empirical observations about the acquisition of data awareness, and general knowledge about data and information technology skills, in youth's everyday online and offline lives (specifically outside of classroom and away from home with family) [33]. The research is specifically focused upon informal settings, negotiated amongst peers and online communities, and in public space where teens bring their own devices (commonly referred to "BYOD") - as is now frequently seen in public libraries throughout the U.S. $[6,18]$.

Hanging out with teens in public libraries is particularly useful for studying how people age with information technology and acquire skills because it is often one of the first sites where teens are exposed to transactional public goods, civic life, infrastructure and public services such as free $\mathrm{Wi}-\mathrm{Fi}$, places to sit, and be exposed to free information resources that they may browse, use, or borrow [10, 19, 34]. Our ongoing study of youth data literacy has three purposes. First, as a means of examining and gathering empirical evidence about young people's current understandings of data.
Second, to pilot library-based programming for informal learning experiences that meet teens' needs and reveal the relationship between social media platforms and the data traces that are created and collected by mobile devices and social media platforms.. And third, to gather qualitative observations about how teens understand data in situ with their own personal technology, and in turn, what it means to be a teen becoming a data citizen, interacting in different networked digital data contexts (what can be referred to as, "data worlds") [31].

With this paper we aim to make a theoretical contribution to current definitions of "data literacy" as it relates to youth. We also report on some of our methodological innovations that that were designed to reveal how teens use wireless technology, understand information infrastructures, and become active, inactive, or departing members of social media platforms. Indeed, using wireless technology and creating data is a form of belonging in contemporary society and has different stakes for youth [12, 29]. With regard to scholarship on learning with digital and social media (DSM), we proffer some ways of studying teens and DSM that aims to deepen our theoretical understandings of knowledge acquisition in informal learning settings [35-36]. We also aim to challenge the information and communication research community to study the relationship between young people's development and the sociotechnical processes of data literacy, which are subject to the rapidly changing digital landscape of personal wireless devices, that deeply affect who and how we learn [14, 21, 23, 33]. While many social scientists have studied college students, transitional knowledge workers, and seniors acquiring data skills and their use of social media platforms such as Facebook or Twitter, there is little research specifically focused upon youth creating data traces with multiple wireless devices-for example, those teens who have standard-issue tablets or laptops in addition to their own personal mobile devices.

Groundbreaking studies on teens and digital media $[11,23,26]$, tend to ask teens about how they use social media platforms (and are social on them) in order to theorize how identity is constructed through use and performance. We build off these studies by focusing on how participants used tools to access data across platforms and learned about these networked collection contexts from the outside in. For example, how do teens understand themselves as "users" or creators of data if we start by asking them what kinds of wireless devices they own, expected to use as students, have knowledge of, and share with their family and friends? By beginning with device ownership (including native mobile operating systems), followed on by skills acquisition and user 
accounts, we can understand the on-the ground use of platforms through their personal devices, we are able to follow teens as they "learn to drive" with these devices, platforms, user profiles, and new forms of networked sociality.

In this paper we explore the question, "What do young people understand about data, creation and management, and the devices they use in their everyday lives?" In the following sections we cover background, related literature, the design and method of the on-going study. We then discuss and interpret findings from a series of semi-structured interviews with young people, ages 11 to 18 , that examined teens' perceptions and general knowledge of data in their lives by way of the mobile devices they own and use. Results suggest that the teens in this study have a number of different interpretations of the nature of data and an awareness of the life cycle of data that is shaped by mobile computing and wireless infrastructures; most found it difficult to connect with data at a personal level in terms of management and storage, while many had nuanced and sophisticated understandings of creating data in platforms and apps with mobile devices having owned and upgraded several times as children and young adults. We argue that the shift to mobile platforms (including operating systems and apps) has a significant influence on how teens understand themselves as data subjects and acquire data literacy skills.

\section{Background}

Contemporary definitions of data literacy come from a number of fields, including information science, data science, business management, learning sciences, and library education. Definitions traditionally rely on skills related to statistics, numeracy, machine learning, quantitative reasoning, and increasingly processing and visualization of data. However, in the past few years, information scholars, librarians, and educators have moved towards broadening the scope of data literacy to include humanistic interpretation, habits of mind, creative expression, and ways of understanding ourselves and digital culture through critical skills that begin with a deep understanding of the data lifecycle. Still, even with broader definitions of data literacy skills, most research and educational initiatives tend to be focused on adults in college or in the workplace [9, 13, 24, 39]. And yet, there is scant evidence of what data literacy means for young people on the cusp of adulthood, who have not yet entered the workforce or higher education and are still untrained in research data management. Teens use digital media tools and social media platforms to consume information, creating, accessing, and curating data at staggering rates [21, 23], but we don't have much empirical evidence of how these contexts help them know themselves or change what it means to be a teen.

Despite the possibilities of creating vast troves of information, little is known about the massive collections of data and its impact on youth today and their development as young adults. The skills and knowledge that youth need to understand the stakes of creating, managing are different than knowledge workers in the $21^{\text {st }}$ century workforce or college students learning how to leverage higher education electronic resources. This gap in the literature that defines and shapes contexts of data literacy learners motivated us to ask what does the term "data" mean in the everyday lives of young people - particularly, what does it mean outside of the classroom. This is an important question for scholars of technology given that the fast-growing trends of young people mediating their lives through digital, networked platforms and the powerful ways that mobile phones are increasingly becoming primary computing devices.

While evidence suggests that there are consequences on adults' habits-from information seeking and filter bubbles to algorithmic bias - these impacts on young people are yet unknown. The ways in which their personal data collections shape their identities and future opportunities have been discussed and speculated $[17,20,31,38,40]$. And further, as primary and secondary education increasingly relies on devices and learning management systems, we have little knowledge of how student data and youth's personal data are related, connected, or intertwined. It's clear that BYOD and mobile device saturation is distributed and arranged across different demographics, locations, and regions $[6,18]$. We find a range of understandings of personal data in teens-a range of peaks and troughs of knowledge according to platform, age, or the idea of a shared family resource or device [5].

\section{Related Literature}

Notions of literacy transform each time there are shifts in information and communication technologies (ICTs). Measurements of literacy have ranged from being able to sign your name, to read the printed words like the newspaper, to finding a book with the card catalog or distinguish between a ".gov" and ".com" website. As ICTs grow, change, even fall out of fashion, expectations of what literacy skills are needed to operate in different worlds, from the workplace to classrooms transform as well. Some researchers have defined data literacy as the skills needed to 
meaningfully consume information in an era of big data and the 24-hour news cycle. These scholars are responding to the contemporary phenomena known as "information overload" primarily as an outcome of news media and information found on the web [24]. The range of data literate skills needed to wade through "info-glut" as it is sometimes called are searching, filtering and processing, producing or creating information, and the ability to synthesize [24]. Effectively searching is not a new information literacy skill, and yet, with search engines and the rise of personalization web tools that create filter bubbles, discerning readers need to understand the architecture of the data they are accessing and exposed to in platforms, in addition to the impact and results of algorithmic sorting [16, 24, 32, 38].

With the rise of fake news and Facebook walls, "filtering" skills are needed for readers to discern and trust information published within and across platforms such as stories posted to Facebook's personalized newsfeed. Recent studies from Common Sense Media and the Knight Foundation after the 2016 election show that teens have high rates of confidence in their filtering abilities, but low rates at correctly identifying fake news, facts, or vetted information [15, 23]. Technologists and engineers have argued that understanding these platforms can be comprehensive, even empowering, if users create more data. For example, Johnson [22] argues that authorial agency through digital self-expression is necessary for understanding data landscapes and online publishing skills through platforms like Wordpress or Medium which involve modularity and infrastructural thinking - thus the process of refinement by consuming and creating data in platforms can lead to the critical skill of synthesis across sources, services, and infrastructure.

Increasingly we see advice about data literacy and empowerment directed towards workers in teams. In their book on data-driven decisions in business marketing and product management, Tunguz and Bien discuss a number of data literacy case studies from tech companies like Facebook and Zendesk [39]. They identify seven principles of data literacy for working in corporate teams that range from SQL, data architecture, data dictionaries (or metadata), a familiarity with case studies and how teams solve data related problems, basic statistical concepts such as sample bias, significance, and confidence intervals; telling stories with data to create an argument (including visualizations), and understanding "actionability" of data analysis to make changes to business operations. Tunguz and Bien describe data literacy in terms of cross-functional teams responding to a new kind of "business intelligence" needed for the big data era. Largely this involves ecological assessments of different stakeholders and understanding the different data pipelines and breadlines in large corporations. By thinking of data literacy in terms of teamwork and empowering small groups of people to make actionable change, the meaning of data literacy through business intelligence allows teams and managers to espouse new management principles most responsive to the big data era.

Two other approaches to data literacy in library and information science (LIS) exist, situated within the skills acquisition and learning environments of academic libraries and higher education. While both are concerned with learning in formal and informal contexts, neither focus on youth or mobile computing with wireless devices. Calzada Prado and Marzal map core data literacy competencies to a traditional information literacy framework that include understanding data, finding and obtaining data, reading, interpreting, and evaluating data, managing data, and finally, using data [13]. Similar to those experts who frame data literacy in terms of strategies for confronting information overload to support an informed user of social media platforms or digital news media, Calzada Prado and Marzal focus on searching, retrieving, vetting and making an argument with information sources by applying data after finding and consuming it.

Library researchers Carlson and Johnston offer an alternative definition in their book, Data Information Literacy: Librarians, Data, and the Education of a New Generation of Researchers which focuses on training undergraduate and graduate students in STEM disciplines [9]. Carlson and Johnson analyze the roles that university research libraries can take in supporting the data management, workflow and curation expertise needed for advanced, specialized science and engineering students need. Working with faculty and graduate students, Carlson and Johnson identified twelve data information competencies that map onto the data lifecycle. These include: discovery and acquisition of data, databases and formats, data conversion and interoperability, data processing and analysis, data visualization and representation, data management and organization, data quality and documentation, metadata and representation, and cultures of practice. While Carlson and Johnston's data information literacy model is one of the only empirical models situated in libraries, the framework and most of competencies have little to do with the creation of data or the agency achieved by understanding personal data collections or owning personal mobile devices that construct an individual's possible data worlds. Instead, 
it largely is focused on scholarly research data management.

Existing orientations to defining data literacy skills and competencies focus on the workplace, working in teams, consuming and vetting data as a consumer who is suffering from information overload, and acquiring tool-based skills related to graduate level research. We argue that the field of data literacy needs to be broadened beyond learning in the academy, acquiring new tactical skills for managing the deluge of data, or reacting to unique data challenges in the workplace. Given that current evidence of youth engagement with digital platforms and mobile devices ownership marks teens, 11-17 as the fastest growing segment of data creators $[18,20]$, there are some gaps in popular accounts of data literacy and the LIS models of core competencies that need to be explored further, which this research aims to address.

\section{Method}

\subsection{Data collection}

This paper includes quotations and self-reported device ownership statistics gathered from 13 exploratory in-person interviews with 22 teens about youth data literacy in the context of library programing for teens, with a focus on mobile devices, social media platforms, and internet skills. For the purposes of this paper, we report specifically from interviews designed to discover how teens define data and how it relates to their experience of using and owning mobile devices that connect to the internet. The interviews were conducted by the research team between December 2016 and March 2017, each lasted between 20-40 minutes. We recruited participants and conducted interviews in three urban library branches of the Carnegie Library of Pittsburgh in teen spaces known as "The Labs". The Labs are teen-only spaces where young people can hang out and work on projects, homework, 3D print, work with film-editing or music recording software, sew or do handcrafts, play board games and video games, and socialize.

We offered teens the option to be interviewed individually or in small groups. The semi-structured interviews followed a theme-driven research protocol focused upon: the meaning and rhetoric of data, data systems and flows, data agency and subjectivity, rights and values, data management skills, and youth views on the role of public library in programming or supporting data knowledge and skills. These themes fit within a larger conceptual structure driving this project, which envisions data as an interplay between collections and community practices. The sample included participants from diverse racial, ethnic, and economic backgrounds. Participant ages ranged from $11-18$, the mean age of participants is 15.3 ; males comprised $64 \%$ of participants, and females comprised $36 \%$. We recruited participants from public libraries serving inner-city populations, primarily of lower socio-economic status in Pittsburgh and we estimate that at least half of the sample comes from underserved populations.

\subsection{Data analysis}

Interviews were transcribed verbatim and then deidentified (e.g., real identifiers were replaced with pseudonyms). The analysis proceeded through five consecutive phases, each phase designed to gradually reduce the data to essential themes associated with teen data literacy, having already imposed a thematic structure upon the interviews. The findings and interview excerpts presented are specifically related to disclosure of device ownership (kind of devices, operating systems, apps, and use-cases) and definitions of data and awareness of its creation, collection or transmission.

\section{Findings}

We began each interview by asking teens what kinds of devices they used that accessed wireless networks and allowed them to create, access, or transmit data. All of our participants owned smartphones, 2 male participants (ages 11 and 17) reported owning a second mobile phone. Figure 1 presents the spread of internet connected devices that the interviewees reported. Saturation of our cohort data matches trends of the near penetration of American adults who own mobile devices (as of 2015, 92\% of Americans own a cell phone) [7, 37]. The Pew Research Center has found that this rise in access is to do with the ownership of mobile devices as the primary force between teens' use of the internet $[25,37]$. Smartphones that connect to next generation networks and WiFi facilitate a shift to data-rich social and media messaging platforms or "apps" that increase the digital traces and data collections that users create across platforms such as Whatsapp, Instagram, Snapchat, Facebook Messenger and Twitter. 
Figure 1. Devices owned by participants.

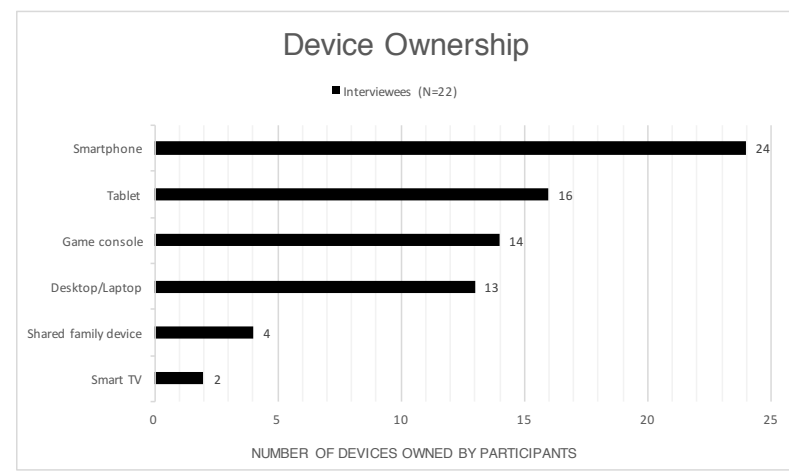

Our survey instrument covered a number of device ownership possibilities, leaving it open ended to the teens. No one reported owning an MP3 player or ebook readers, though $67 \%$ interviewees reported owning a game console or having given a gaming station to a younger sibling. Game console ownership remains consistent with national trends among American teens, though many participants mentioned using their phones as their primary gaming device. All of our interviewees reported having access to school issued devices to complete homework or in-class exercises. We found that students were more likely to have a second personal computing device at home if they had a school issued laptop (42\%). While all interviewees reported having access to school laptops or tablets for class work, only $67 \%$ of interviewees reported a take-home school issued devices that ranged from iPads to Chromebooks. Moreover, when asked if they borrowed or used technology from the library, $57 \%$ of participants with school issued tablets were more likely to borrow a laptop from the library to complete homework (as opposed to using the tablet provided their school).

Teens in the study reported owning multiple mobile and wireless devices beginning in middle school. Interview data that we collected confirmed that devices are often shared in families, with an "at home" communal iPad or gaming consoles that have been passed down through sibling sets.

Adam (age 11): Well, devices sort of run in our family [...] I have a phone with me right now. I have an iPad Mini at home. I have another iPad at home that was sort of passed down. And I have my really old phone.

Adam reported having two cell phones that had different functions - one for calling and the other for messaging and browsing the internet. A number of participants described dedicated devices for particular kinds of uses, such as doing homework with a schoolissued laptop, using Facebook on a home device, or gaming with a device shared with a sibling. Different devices for different use cases was usually related to limited storage on mobile devices, higher processing power for gaming on laptops, or accessing content-rich sites like Facebook or YouTube through a browser instead of their mobile apps. For example, Ginny borrows her sister's tablet for watching video but her brother's laptop for gaming.

Ginny (age 16): I'm 16, I use my phone, my laptop, and sometimes my sister's iPad. Or my brother's computer.

Interviewer: What kind of computer does [your brother] have?

Ginny: I think it's an ASIS, maybe? I'm not sure [...] It's more of his gaming computer. I only play games on it sometimes, though.

When asked about the experience of owning a device for more than a few years, participants often described cycles of device upgrade and the risks of platform lock-in with mobile phones. For example, Fred (age 16) reported having owned a number of iPhones since the fifth grade beginning with the iPhone $4 \mathrm{~s}$ up to the iPhone 6. Some participants described ownership in terms of costs, ranging from data plans with different mobile service providers, to free and paid apps, even the devices themselves and whether the upgrade cycle kept them from repair or upkeep. Isaac for example, was attentive to the low cost-benefit uses of native applications like FaceTime that the iOS platform is known for over the utility of an Android mobile phone that "works" more like a computer and is easier to replace.

Interviewer: Have you always been an Android user? Isaac (age 17): Yeah, because financially, I wasn't... So we started out on 50 dollar phones from Cricket and they were Androids. So I'd use those, I got used to the Android and everything like that, so I figured, for the iPhone... It's not like I'm going to FaceTime anybody, and that's the only thing that iPhones have above me, is video chatting, and I'm not using that, so why not get this? [...] And I feel as if Androids are more so computers. They work as computers while I think Apples are just phones. That's my personal opinion.

After discussing owning mobile devices and personal computers, we asked each of our teen participants to define data and provide examples of it in their lives. Interviewees defined data broadly in three different ways: as quantitative numbers that can be processed and structured as evidence of some action; data-as-access to the internet that has a shared 
value as a limited resource depending on which devices is used on what wireless network; and as 'stuff' that moves through networks, usually mobile media that is transmitted through apps or social media, digital traces that are easily spreadable and without clear ownership expectations but often contained or taking up storage memory on smaller devices.

Data was often described as "numbers" with evidence of action, while a smaller segment of older teen participants (aged 16-18) reported that data were quantitative or numerical representations of information in structured documents such as spreadsheets or report cards.

George (age 13): I feel that data is a quantitative measurement... it shows meaningful values and it is referenced a lot in research. I do a lot of science fairs and usually the winning projects have some type of significant data collection. It'll have a very nice table or graph or multiple graphs."

Participants, like George who explained data as statistics or measurement often used visual representations such as charts or graphs in spreadsheets to explain processed data, while only one interviewee described "data" as computer code. Several participants described data in terms of numbers being presented as evidence, a visualization that supports an argument which may process 'raw numbers' and placed this kind of data in math or science classes, or competing in science fairs.

Early on in our conversations with teens about data and wireless device ownership, we learned that they think of data as access to the internet particularly through mobile web browsing and social media apps. Our interviews suggested that teens think of data as a limited resource that has monetary value and sharing expectations amongst small groups. All of the interviewees owned smartphones and commented on how they accessed wireless or mobile networks in a variety of ways, including using the library's public WiFi. In one-on-one interviews and group discussions, participants often explained data in terms of data plans through their mobile service providers and primarily part of shared family plans (where multiple cell phones and numbers have access to an allotment of data access and transmission rates with certain speeds per billing cycle). Smartphones with apps use internet rich connections to access Facebook or upload Instagram posts, and thus the "data usage" has shared value implications for teens as family members because data transmission rates are a limited resource on a group data plan. Thus 'it pays' to switch mobile phones from a $3 \mathrm{G}$ mobile network to a public WiFi connection or into airplane mode to conserve and save data when you share or a have a limited data plan. For instance, many participants explained the value of this limited resource if they had a brother or sister who gamed on their personal devices and "ate up" access on the family data plan. Unlimited data plans are touted as unique to teens. Even with this savvy understanding of data as access to rich-internet content, younger teens had trouble distinguishing the difference between accessing mobile broadband internet and switching a mobile device to a home or public WiFi network and would explain varieties of faster internet access speeds in terms of accessing "heavy" mobile media at home, such as watching Netflix or playing networked games.

Once interviews shifted from asking participants what data is to where it lives, participants began to use digital traces created with mobile phones and apps to explain transmission, collections, privacy, ownership and agency over data. Interviews suggest that teens think of data as easily spreadable and public facing, often collected by the government or advertisers. Some older teens described how data is already collected for secondary use applications by the government, corporations for advertising, college admissions, or for ad-targeting in platforms based on their use habitsand connected this to their sense of self or their online personas. For example, Kevin (age 14) explained that Amazon tracked shopping habits over time, but also expressed a temporal sense of how data can be collected and used in different contexts and follow you over time, saying: "[...] if you get a bad grade, it usually sticks with you for a while, and you can see all your grades and they, like...Colleges look all the way back." Hannah bemoaned the frequency with which specific kinds of online information seeking lead to targeted ads that don't seem to change, and follow her across platforms even as she has aged:

Hannah (age 18): For me, I'll look up something like Planned Parenthood or something, and now I can't get away from any sort of birth control ad. It's just following me forever. I'm sure they're right, but I don't think I need four Nexplanon ads in my day.

In addition to traces of online behavior, mobile media created and shared by teens in messaging platforms was considered easily transmitted, collected and discoverable but not easily controlled by the teens themselves. Many commented on creating mobile media with their mobile phones at earlier in high school but then having an experience that lead them to be more mindful or cautious data's circulation and reception. Participants understood that apps and online services could access location information and other kinds of data stored on their mobile devices, but few thought of this through the lenses of privacy, rights, or 
ownership. As with single function devices, teens often reported distinctions between useful, social, or creative apps.

Emma (age 14): I have a lot of apps. I have a lot of photo editing apps, then I have my school-related apps, and my anime-related apps, and I have games, my shopping apps, my music apps, my social media apps.

Interviewer: Are they all organized in files?

Emma: Yeah, they're organized by category. And they're alphabetized.

Three older teen boys aged 16-17 reported not using social media on their phones but simply using apps to play games and listen to music through streaming services. Danny (age 17) reported a preference for pragmatic apps that supported media consumption and information seeking on his phone over ones that support the creation of mobile media, "I have various music apps. I have my email. I use practical things. I have bird identification apps [...] Sort of random things, but just useful things, really." Male participants often described information seeking apps (stock forecasting, weather, sports statistics, IMDB) as being useful but admitted to avoiding using social media apps or using them reluctantly.

\section{Discussion}

Three technology trends have occurred in the last decade that have driven the use of social media and mobile apps and the near complete penetration rates of mobile device ownership with American teens. First, the rise of broadband connections including mobile networks and Wi-Fi coverage throughout the world in public and private spaces. Second, this mobile connectivity has coincided with the proliferation of low cost of personal, mobile devices like cell phones, tablets, and later smartphones. Mobile phones that can connect to wireless networks have transformed what the internet means for teens of today in terms of accessibility, use, and even habituation of services. These mobile devices have also influenced the ways users make sense of both mobile and personal computing operating systems, files, and data, digital traces, reading and attention. Increasingly, teens access the internet and internet based services through mobile apps that may stream content such as Spotify or Youtube. The Pew Internet Project recently reported that $70 \%$ of teen internet users have sought advice from someone else at some point about how to manage their privacy online if they download apps [28].

Teens lives are saturated with technology, platform services, and user accounts that is pervasive, portable and persistent in ways that have yet to be studied and understood. This context has implications for new learners and the future of learning processes, in addition to providing literacy skills and technical education. We argue that presently young peoples' data worlds are being influenced by their use and ownership of mobile devices. Future information ecosystems and learning ecologies will be shaped by ownership and authorship of mobile data traces, even now we are seeing the ascendency of seeking and discovery transforming - it is frequently offloaded and funneled through intelligent personal assistants such as Siri, Google Assistant, or Alexa. Our findings found that teens have different priorities and needs from traditional library services that maybe offered to patrons with personal computers. Moreover traversing the space between mobile platforms that support streaming data to the file-based directory structure of personal computer data architecture is vast and not clear for youth. In the interviews, teens often commented on the experience of limited storage on mobile devices and tensions between streaming services versus, processing power, and local storage. This suggests that understanding the traditional data lifecycle as creation, management, curation, preservation of digital data that is formatted in files or databases is radically disrupted by mobile computing and wireless devices like tablets. Based on these broad findings and the impact of mobile devices on youth data literacy in these interviews, it is clear we need to develop new frameworks for explaining the networked, distributed and multiple device and upgrade cycle into present-day understandings of the data management skills.

We take as our starting point then that young people's development is a social process and that becoming an American teen in 2018 involves owning wireless technologies, creating user accounts, and interacting with mobile media - a variety of different kinds of data worlds. By situating this investigation of youth data literacy within the context of personal data management and early contexts of use and technology adoptions, as we do in our project, we give teen participants a meaningful frame as they acquire data skills, including creating, understanding and curating the data collections in their increasingly networked lives. While there is a broad literature to information literacy and digital youth, there is little research that explicitly focuses on youth data literacy. It's clear that the use of mobile applications and mobile devices leaves traces of digital data and has a new form of participatory culture in a networked society. Further, this form of belonging and networked engagement is increasingly a necessary part of becoming and belonging in youth peer culture $[3-4,11,25,27,30]$. 
Still research on teen's awareness of data is limited, and few scholars have examined youth's data worlds through the traces created by the devices they own or the mobile media that they create-data footprints. There is a strong body of evidence that reveals young people do have notions of privacy, ownership, and digital data by way of their online practices and being social online [28, 29]. Nevertheless, we need more nuanced understandings of how they make sense of data and become aware of it, so that we can shape how they acquire data skills and participate in a lifetime of personal data management.

\section{Future Work}

This research begins with the idea that all people, including teens, have rights to access, own, and understand data created by and about them. Young folks can and do play a role in the construction of their own data subjectivity by understanding their digital dossiers and data footprints. Those who study, teach, and engage with teens can learn from these findings if we begin to turn our attention towards teens' data awareness and dispositions that relate to a number of civic, legal, and ethical matters. We've addressed some of these issues in the findings reported here, by reporting on how young people understand themselves as data subjects, technology users, and devices owners, each of which are all facets of data literacy including the capacities to understand, create, and manage personal data collections. Using the coding structure developed and workshop interventions designed in this first phase of this project, we continue our exploration into young peoples' interactions with data, focusing in greater detail data management skills acquisition, and expanding our scope of how participants learn how data effects their personal privacy and agency as young adults. Our hope is that this work that examines how teens perceive data as part of device ownership and social media interaction in the contexts of their everyday lives, specifically as they shift to primarily using mobile operating systems, will provide early evidence and action for developing data programming and information services for young people in informal learning contexts and public spaces such as libraries and community centers.

\section{References}

[1] A. Acker \& L. Bowler, "What is Your Data Silhouette? Raising Teen Awareness of their Data Traces in Social Media," in SMSociety '17 2017 International Conference on Social Media \& Society, 2017.
[2] A. Acker, Born Networked Records: A History of the Short Message Service Format, University of California, Los Angeles, 2014.

[3] D. E. Agosto, J. Abbas \& R. Naughton, Relationships and social rules: Teens' social network and other ICT selection practices, Journal of the American Society for Information Science and Technology, 63:6, pp. 1108-1124, 2012.

[4] J. Ahn, The effect of social network sites on adolescents' social and academic development: Current theories and controversies, Journal of the American Society for information Science and Technology, 62:8, pp, 1435-1445, 2011 .

[5] M. Alper, Giving voice: Mobile communication, disability, and inequality, Cambridge, MIT Press, 2017.

[6] American Library Association, K. S. Rosa, ed. The State of America's Libraries 2017: A Report from the American Library Association, 2017,

http://www.ala.org/news/sites/ala.org.news/files/content/Stat e-of-Americas-Libraries-Report-2017.

[7] M. Anderson, Technology Device Ownership: 2015, PEW Research Center, Internet, Science \& Technology, 2015, http://www.pewinternet.org/2015/10/29/thedemographics-of-device-ownership.

[8] C. L. Borgman, J. C. Wallis, M. S. Mayernik, \& A. Pepe, "Drowning in data: digital library architecture to support scientific use of embedded sensor networks," in Proceedings of the 7th ACM/IEEE-CS joint conference on Digital libraries, 2007, pp. 269-277.

[9] J. Carlson \& L. R. Johnston (Eds), Data Information Literacy: Librarians, Data, and the Education of a New Generation of Researchers, Purdue Information Literacy Handbooks, Ashland, Purdue University Press, 2015.

[10] E. S. Deahl, E. Better the data you know: Developing youth data literacy in schools and informal learning environments (Masters Thesis), Cambridge, Massachusetts Institute of Technology, 2014, http://dx.doi.org/10.2139/ssrn.2445621.

[11] d. boyd, It's complicated: The social lives of networked teens, New Haven, Yale University Press, 2014.

[12] d. boyd, "Why youth (heart) social network sites: The role of networked publics in teenage social life," in MacArthur foundation series on digital learning-Youth, identity, and digital media volume, 2007, pp. 119-142.

[13] J. Calzada Prado \& M. Á. Marzal, Incorporating Data Literacy into Information Literacy Programs: Core Competencies and Contents, Libri, 63(2), 123-134, 2013.

[14] S. W. Campbell, R. Ling, \& , J. B. Bayer, "The structural transformation of mobile communication: 
Implications for self and society" in Media and social life, 2014, pp. 176-188.

[15] Common Sense Media, News and America's Kids: How Young People Perceive and Are Impacted by the News, 2017, https://www.commonsensemedia.org/research/newsand-americas-kids.

[16] K. Crawford, Can an algorithm be agonistic? Ten scenes from life in calculated publics, Science, Technology, \& Human Values 41.1, pp. 77-92, 2016.

[17] B. Friedman \& H. Nissenbaum, Bias in computer systems. ACM Transactions on Information Systems (TOIS), 14(3), pp. 330-347, 1996.

[18] M. L. Hartman, S. Hughes-Hassell, and K. D. Kumasi, The future of library services for and with teens: A call to action. Linda W. Braun, ed., Young Adult Library Services Association, 2014.

[19] K. M. Hofman, M. Subramaniam, S. Kawas, L. Scaf, \& K. Davis, Connected libraries: Surveying the current landscape and charting a path to the future, College Park, MD; Seattle, WA, The ConnectedLib Project. 2016.

[20] L. D. Introna \& H. Nissenbaum, Shaping the Web: Why the politics of search engines matters, The information society, 16(3), pp. 169-185, 2000.

[21] M. Ito, S. Baumer, M. Bittanti, R. Cody, B. Herr Stephenson, H. A. Horst, P. G. Lange et al., Hanging out, messing around, and geeking out: Kids living and learning with new media, Cambridge, MIT press, 2009.

[22] C. A. Johnson, The Information Diet: A Case for Conscious Consumption, O'Reilly Media, Inc., 2015.

[23] Knight Foundation, Understanding Snapchat: Media, Politics and the 2016 Election, John S. and James L. Knight Foundation, 2017, https://kf-site-

production.s3.amazonaws.com/publications/pdfs/000/000/23 7/original/ENP-Snapchat-Survey_final.

[24] T. Koltay, The bright side of information: ways of mitigating information overload, Journal of Documentation, 73.4, 2017.

[25] A. Lenhart, Teens, Social Media and Technology Overview 2015, PEW Research Center. Internet, Science \& Technology, 2015,

http://www.pewinternet.org/2015/04/09/teens-social-mediatechnology-2015.

[26] S. Lincoln \& B. Robards, Editing the project of the self: sustained Facebook use and growing up online, Journal of Youth Studies, 20.4, pp: 518-531, 2017.

[27] S. Livingstone, Developing social media literacy: How children learn to interpret risky opportunities on social network sites, Communications: The European Journal of Communication Research, 39:3, pp. 283-303, 2014.

[28] M. Madden, A. Lenhart, S. Cortesi \& U. Gasser, Teens and Mobile Apps Privacy, PEW Research Center, 2013, http://www.pewinternet.org/2013/08/22/teens-and-mobileapps-privacy.

[29] A. E. Marwick \& d. boyd, Networked privacy: How teenagers negotiate context in social media," New Media \& Society 16.7, pp. 1051-1067, 2014.

[30] C. L. Moore, A study of social media and its influence on teen information seeking behaviors, The Serial Librarian, $71: 2,2016$, DOI $=10.1080 / 0361526 X .2016 .1209452$.

[31] A. Mulder, J. Brouwer, M. Delanda, A. Appadurai, S. Plant, G. Legrady, A. Versluis et al. Information is Alive: Art and Theory on Archiving and Retrieving Data, V2/NAi Publishers, Rotterdam, 2003.

[32] E. Pariser, The filter bubble: How the new personalized web is changing what we read and how we think. New York, Penguin Press, 2012.

[33] L. Robinson \& D. Bawden, 'The story of data': a sociotechnical approach to education for the data librarian role in the CityLIS library school at City, University of London, Library Management, 2017, DOI 10.1108/LM-012017-0009.

[34] K. W. Schaie (Ed.), Social structures and aging. New York: Springer, 2000.

[35] K. A. Scott \& K. Clark (Eds.), Digital Engagement for Urban Youth: From Theory to Practice [Special Issue], Urban Education, 48(5), 2013.

[36] K. A. Scott \& M. White, COMPUGIRLS' Standpoint: Culturally responsive computing and its effect on girls of color, Urban Education, 48(5), 2013, pp. 657 - 681.

[37] A. Smith, "The Smartphone Difference," Pew Research Center, 2015,

http://www.pewinternet.org/2015/04/01/us-smartphone-usein-2015/.

[38] L. Sweeney, Discrimination in online ad delivery. Communications of the ACM, 56(5), pp. 44-54, 2013.

[39] T. Tunguz \& F. Bien, Winning with Data: Transform Your Culture, Empower Your People, and Shape the Future, Hoboken, John Wiley \& Sons, 2016.

[40] J. Zang, K. Dummit, J. Graves, P. Lisker, \& L. Sweeney, Who knows what about me? A survey of behind the scenes personal data sharing to third parties by mobile apps. Technology Science, 30, 2015 